\title{
Los proyectos prácticos de la Cátedra Inditex-UDC de Sostenibilidad como experiencia innovadora en el ámbito de la educación para la sostenibilidad
}

Mato-Santiso, Vanessa'; Rey-García, Marta ; Salido-Andrés, Noelia²; Taboadela-Álvarez, Obdulia $^{3}$

\author{
${ }^{1}$ Cátedra Inditex-UDC de Sostenibilidad \\ ${ }^{1,2}$ Facultad de Economía y Empresa, Universidade da Coruña \\ ${ }^{3}$ Facultad de Sociología y Ciencias de la Comunicación, Universidade da Coruña

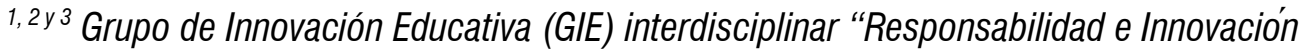 \\ Social + Sostenibilidad $(R I S+S) "$
}

\section{RESUMEN}

La Cátedra Inditex-UDC de Sostenibilidad nace como un espacio de reflexión -formación, investigación y divulgación- en el ámbito de la sostenibilidad, la responsabilidad e innovación social. El objetivo de esta comunicación es analizar los proyectos prácticos de esta cátedra en colaboración con entidades como metodología de innovación educativa y transferencia de conocimiento, basada en el aprendizaje colaborativo entre seis actores diferentes: la dirección académica, los alumnos, los representantes de las entidades colaboradoras, el tutor metodológico, los antiguos alumnos que mentorizan los proyectos y los miembros del tribunal. Para ello, se hace especial hincapié en los retos que supone su desarrollo, se detallan las acciones de mejora continua adoptadas y se evalúan tanto los resultados de aprendizaje como los resultados de implantación de las recomendaciones de los alumnos en las entidades colaboradoras. Hasta el momento, estos proyectos en colaboración se traducen en un modelo exitoso de aprendizaje para los alumnos, y en transferencia de conocimiento a las entidades colaboradoras, que obtienen ideas y recomendaciones innovadoras para resolver necesidades reales e inmediatas. 
PALABRAS CLAVE: Innovación educativa, Sostenibilidad, Aprendizaje colaborativo, Transferencia de conocimiento, Cátedra Inditex-UDC de Sostenibilidad

\section{CITA RECOMENDADA:}

Mato-Santiso, V.; Rey-García, M.; Salido-Andrés, N.; Taboadela-Álvarez, 0. (2019): Los proyectos prácticos de la Cátedra Inditex-UDC de Sostenibilidad como experiencia innovadora en el ámbito de la educación para la sostenibilidad. En De la Torre Fernández, E. (ed.) (2019). Contextos universitarios transformadores: construíndo espazos de aprendizaxe. III Xornadas de Innovación Docente. Cufie. Universidade da Coruña. A Coruña (pág. 359-374).

DOI capítulo: https://doi.org/10.17979/spudc.9788497497121.359

DOI libro: https://doi.org/10.17979/spudc.9788497497121

\section{ABSTRACT}

The Inditex-UDC Chair of Sustainability emerged as a space for reflection -training, research and dissemination- in the field of sustainability, responsibility and social innovation. The objective of this communication is to analyze the projects of this chair in collaboration with entities as educational innovation and knowledge transfer methodology, based on the collaborative learning among six different actors: Academic Direction, students, representatives of the collaborating entities, the methodological tutor, alumni who mentor the projects and the members of the Academic Tribunal. Along these lines, the challenges encountered in its development are highlighted, the continuous improvement actions adopted are detailed, and the results of the learning and results of the implementation of recommendations from students on the collaborating entities are evaluated. So far, these collaborative projects translate into a successful learning model for students, and knowledge transfer to collaborating entities, which obtain innovative ideas and recommendations to solve real and immediate needs.

KEY WORDS: Educational innovation, Sustainability, Collaborative learning, Knowledge transfer, InditexUDC Chair of Sustainability 


\section{INTRODUCCIÓN}

Con una población mundial creciente y recursos naturales limitados es imprescindible aprender a vivir de forma sostenible. En 1987, la Comisión Mundial de Medio Ambiente y Desarrollo de las Naciones Unidas definía el desarrollo sostenible como el "desarrollo que satisface las necesidades del presente sin comprometer la capacidad de las generaciones futuras para satisfacer sus propias necesidades" (WCED, 1987, p.37). Más recientemente, Naciones Unidas acordó en 2015 diecisiete Objetivos de Desarrollo Sostenible (ODS) con el objetivo de marcar una agenda universal para el desarrollo sostenible, protegiendo al planeta y preservando recursos para las futuras generaciones. Esta agenda de desarrollo sostenible une a los gobiernos, sector privado, sociedad civil y al resto de ciudadanos en torno a una meta común, que va desde el acuerdo sobre los objetivos hasta su implementación y logro (Naciones Unidas, 2018). Teniendo esto en cuenta, la UNESCO tiene como objetivo mejorar el acceso a una educación de calidad en el ámbito de la sostenibilidad, para transformar la sociedad al orientar la educación hacia la adquisición de los conocimientos, habilidades, valores y comportamientos necesarios para lograr un desarrollo sostenible (UNESCO, 2018).

En este contexto nace la Cátedra Inditex de Sostenibilidad de la Universidade da Coruña (UDC), resultado del acuerdo de colaboración firmado en 2010 entre ambas instituciones. Contando con el apoyo y la coordinación del Consello Social de la universidad coruñesa, la Cátedra Inditex-UDC de Sostenibilidad (denominada Cátedra Inditex-UDC de Responsabilidad Social hasta el curso 2018/2019) surge como un espacio de reflexión comunitaria, formación académica e investigación aplicada sobre las distintas dimensiones de la sostenibilidad y la responsabilidad e innovación social. Con el objetivo de adaptarse a un nuevo escenario caracterizado por la globalización, el multiculturalismo y la revolución tecnológica, entre otros factores, este producto formativo apuesta claramente por la mejora continua y la actualización permanente, lo que permite ajustar programas y contenidos a las demandas del mercado (Salido-Andrés et al., 2014; Espinosa et al. 2006). Además, el trabajo en equipo e 
interdisciplinar es una estrategia innovadora para dar respuesta a los problemas múltiples y complejos a los que se enfrenta la sociedad (Fernández, 2002).

El presente trabajo tiene como objetivo mostrar cómo la realización de proyectos prácticos por Ios alumnos del Curso de Especialización en Sostenibilidad e Innovación Social (CESIS) de la Cátedra Inditex-UDC de Sostenibilidad, en colaboración con organizaciones de distintos ámbitos, constituye un modelo de innovación educativa basado en el aprendizaje colaborativo entre seis actores diferentes: 1) la dirección académica, 2) los alumnos, 3) los representantes de la entidad colaboradora, 4) el tutor metodológico, 5) los antiguos alumnos que mentorizan los proyectos y 6) los miembros del tribunal que los valoran en sesión pública.

\section{LOS PROYECTOS PRÁCTICOS DE LA CÁTEDRA INDITEX-UDC DE SOSTENIBILIDAD}

\subsection{EN QUÉ CONSISTEN Y PRINCIPALES RETOS}

El Curso de Especialización en Sostenibilidad e Innovación Social (CESIS) de la Cátedra Inditex-UDC de Sostenibilidad, nace con el objetivo de formar a profesionales y recién titulados de diversas disciplinas en temáticas clave para avanzar en el desarrollo sostenible de las sociedades y la responsabilidad social de las organizaciones. Esta formación de posgrado trata de manera transversal, multidisciplinar y práctica, la sostenibilidad y la responsabilidad e innovación social de administraciones públicas, empresas y entidades no lucrativas, poniendo el foco en las iniciativas más innovadoras que sean capaces de crear conjuntamente valor económico, social y medioambiental.

Durante las cuatro primeras ediciones de esta formación de posgrado, los proyectos prácticos consistieron en emitir propuestas de mejora en el ámbito de la Responsabilidad Social Universitaria (RSU) en la UDC (curso 2011/12), diseñar un cuadro de mando de indicadores de RSU para testarlo en las universidades españolas (curso 2012/13), y analizar el estado de la responsabilidad e innovación social en un sector de actividad, a través de la comparación de

5 organizaciones, y presentar recomendaciones para la mejora de su desempeño en esta materia (cursos 2013/14 y 2014/15). Pero, a partir de la quinta edición del posgrado (curso 
2015/16), surge la idea de la realización de proyectos prácticos en colaboración desde la Dirección académica de la Cátedra para dar respuesta a la necesidad de transferir conocimiento en materia de sostenibilidad, responsabilidad e innovación social a organizaciones de diversa índole. Estas solicitaban colaboración para obtener ideas sobre cómo resolver problemáticas o necesidades concretas.

Los proyectos prácticos en colaboración consisten en la aplicación, por parte de los estudiantes, de los conocimientos que van adquiriendo a lo largo del curso, para dar respuesta a necesidades reales en materia de sostenibilidad e innovación social a las que tienen que hacer frente organizaciones de nuestro entorno. Estos proyectos se desarrollan en equipos de trabajo de 405 alumnos, conformados según sus perfiles y la temática concreta de cada proyecto. Además, cada equipo de trabajo cuenta con el apoyo y orientación de un tutor metodológico, un mentor (que consiste en un antiguo alumno/a del CESIS que aconseja y facilita las dinámicas de trabajo en equipo y la interlocución con la entidad colaboradora), así como un representante de la organización colaboradora.

Este nuevo enfoque en la realización de los proyectos implica algunas ventajas para los estudiantes, como pueden ser la fácil obtención de información relevante para el desarrollo del proyecto (proporcionada por los representantes de las entidades colaboradoras), mayor aprendizaje al tener la oportunidad de aplicar de forma práctica sus conocimientos, y el contacto que establecen con organizaciones de nuestro entorno, lo que puede derivar en colaboraciones futuras 0 incluso en relaciones contractuales.

Entre los principales retos que han sido detectados en la puesta en marcha de este modelo de innovación educativa y transferencia de conocimiento, se encuentran los siguientes:

1. Alcanzar un equilibrio a la hora de definir los objetivos específicos de cada proyecto, entre los conocimientos mínimos que deben adquirir los alumnos y el cumplimiento de las expectativas de las entidades colaboradoras.

2. La óptima aplicación práctica de los conocimientos que los alumnos van adquiriendo sobre la realidad de una organización. 
3. Superar las dificultades con las que se encuentra cada equipo de trabajo a la hora de desarrollar el proyecto (organización interna, reparto de tareas, comunicación con el equipo de apoyo, etc.).

\subsection{OBJETIVOS Y METODOLOGÍAS ACTIVAS EMPLEADAS EN LOS PROYECTOS PRÁCTICOS}

El proyecto práctico tiene como objetivo principal elaborar un plan de acción para la implementación de actuaciones concretas que den respuesta a los objetivos específicos acordados con cada entidad colaboradora, ajustando su desarrollo a los recursos de los que dispone la organización. Es decir, la propuesta de plan de acción planteada tendrá en cuenta tanto la disponibilidad de recursos de la entidad colaboradora para ejecutarlo (tiempo, dinero y personal), como los requisitos académicos mínimos que dicho proyecto ha de cumplir para ser evaluado por el tribunal. Más concretamente, con esta innovadora experiencia educativa se pretenden alcanzar los siguientes objetivos: 1) la realización de un trabajo de carácter académico, no de consultoría 0 de opinión, que permita a los alumnos adquirir las capacidades necesarias para su desempeño profesional en el ámbito de la sostenibilidad y la innovación social; 2) conocer y manejar las metodologías y herramientas comúnmente aceptadas en esta área; y 3) aprender a trabajar en equipo.

Cada proyecto, dividido en tres o cuatro entregables intermedios, deberá constar de un soporte teórico-conceptual y demostrar el dominio del lenguaje específico en el ámbito de la sostenibilidad, responsabilidad e innovación social. El análisis y propuesta de plan de acción deberán estar debidamente argumentados y apoyados en metodologías comúnmente aceptadas en esta área. Esto permitirá la identificación de las mejores prácticas, entre las desarrolladas por la propia entidad colaboradora y sus grupos de interés clave, en relación a los asuntos más materiales o relevantes para el cumplimiento de los objetivos fijados para cada proyecto. 
A continuación, se describen las metodologías innovadoras que se utilizan a lo largo del desarrollo de los proyectos prácticos para aportar a los alumnos los conocimientos necesarios para la correcta realización de cada entregable:

- Sesiones participativas en las que se aplica el método del caso, que consiste en la resolución de problemas reales en el aula: detectar problemas existentes, discutir diferentes enfoques para su resolución, y evaluar las posibles consecuencias de cada una de las soluciones alternativas alcanzadas. Esta metodología se desarrolla en tres etapas distintas: 1) El estudio individual del caso para alcanzar individualmente posibles soluciones; 2) Trabajo en equipo, discutiendo en equipo los planteamientos individuales; y 3) Sesión general o plenaria, guiada por el docente, para lograr un aprendizaje colaborativo basado en las conclusiones y lecciones aprendidas a lo largo del proceso (IESE, 2018).

- Tutorías para explicar los contenidos mínimos y fases del proyecto y resolver posibles dudas. A las tutorías asisten el tutor/a, los mentores, los alumnos y, en algún caso, representantes de las entidades colaboradoras (normalmente asisten a la primera tutoría exclusivamente).

- Talleres monográficos para el aprendizaje práctico en profundidad de algunos conceptos básicos en el marco del proyecto, como pueden ser la identificación de grupos de interés, la elaboración de un análisis de materialidad o la medición del impacto social.

- Visitas a las entidades colaboradoras $\mathbf{y} / \mathbf{0}$ charlas informativas, con el objetivo de conocer en mayor medida el funcionamiento y el día a día de la organización para ajustar el proyecto a la realidad de la misma.

\subsection{ACTORES INVOLUCRADOS EN ESTE MODELO DE INNOVACIÓN EDUCATIVA}

Existen diferentes tipos de actores que están implicados en esta experiencia innovadora de transferencia de conocimiento, basada en el aprendizaje colaborativo, pero destacan seis: 1) dirección académica del CESIS, 2) alumnos, 3) representantes de las entidades colaboradoras, 4) el tutor/a, 5) los mentores y 6) el tribunal académico que juzgará los 
proyectos prácticos en la etapa final. A continuación, explicaremos en profundidad las distintas funciones y responsabilidades que tienen que asumir cada uno de estos actores principales.

- Dirección académica del CESIS: es la responsable, en primer lugar, de fijar los objetivos generales de los proyectos prácticos de cada edición (p.ej. analizar la transparencia en la web, elaborar un cuadro de mando, elaborar un plan de acción, preparar un caso docente, etc.). Además, se encarga de las siguientes tareas:

1. Seleccionar un conjunto de proyectos diversos y relevantes, a partir de las solicitudes de colaboración recibidas de terceros 0 de las propuestas realizadas desde la propia Cátedra.

2. Consensuar con las entidades colaboradoras los objetivos específicos que se deben alcanzar en cada proyecto.

3. Conformar equipos de alumnos diversos y multidisciplinares. Es decir, se intenta que en cada equipo haya personas de distinto género, distintas edades (junior $<25$ años; pre-senior entre 25-35 años; y senior >35 años), y con formación y experiencia en distintas áreas de conocimiento.

4. Asignar equipos de alumnos a los distintos proyectos en base a varios criterios, entre ellos que cada miembro pueda aportar algo al proyecto en cuestión, y viceversa, que el proyecto pueda aportar valor a cada alumno para su desarrollo profesional.

- Alumnos: Cada proyecto se asigna a un equipo de trabajo formado por 405 alumnos del CESIS. Los alumnos, durante el desarrollo de los proyectos, tienen las siguientes responsabilidades:

1. Elaborar los proyectos prácticos en tiempo y forma, respetando el calendario de fechas de los entregables y el formato y requisitos mínimos exigidos para su defensa ante el tribunal.

2. Respetar a los interlocutores para centralizar las comunicaciones y utilizar los canales 0 vías de comunicación establecidas en cada caso. 
3. Fomentar la cooperación y colaboración en el equipo (realizar un reparto equitativo de las tareas, debatir la toma de decisiones, resolver posibles conflictos que pudieran surgir, etc.).

4. Asistir a las tutorías plenarias, talleres prácticos, así como a las demás reuniones 0 sesiones a las que sean convocados.

- Representantes de las entidades colaboradoras: Las entidades colaboradoras de estos proyectos deberán seleccionar, al menos, a un miembro de su organización para que actúe como interlocutor/a del proyecto asignado, y cuyo rol principal es el de intermediario entre la entidad colaboradora y el equipo que desarrolla el trabajo, proporcionando los datos e información necesarios para el desarrollo del proyecto. Este representante debe asumir el compromiso de atender en tiempo y forma las necesidades de información de los equipos para el correcto desarrollo del trabajo dentro del calendario establecido. Estos intercambios de información entre los representantes y los equipos, que pueden ser presenciales o virtuales, se centralizarán siempre a través de los mentores, y no de forma directa, para tratar de coordinar las peticiones. Por tanto, teniendo esto en cuenta, las principales tareas de los representantes de entidades colaboradoras serían las siguientes:

1. Definir los objetivos específicos del proyecto en coordinación con la dirección académica.

2. Preparar un dossier para el equipo con información relevante sobre la organización, que incluya las principales características de la organización (tipo de organización, antigüedad, misión, número de empleados, presupuesto anual, etc.), así como algunos ejemplos de sus principales competidores y organizaciones comparables.

3. Sugerir y planificar sesiones de formación, reuniones, visitas a la entidad o grupos de interés recomendables para obtener una mayor comprensión de los objetivos específicos que se persiguen con cada proyecto. 
4. Proporcionar comentarios y recomendaciones sobre cada entregable para alinear el trabajo final a los objetivos de la entidad, quedando las decisiones definitivas del proyecto en manos de los equipos.

- Tutor/a metodológico: El/la tutor/a es un antiguo estudiante del posgrado, con experiencia en investigación, cuya principal tarea es hacer el seguimiento de los proyectos prácticos. Entre sus tareas se encuentra el asesoramiento metodológico a los equipos en cada una de las fases de elaboración del proyecto, la coordinación de los mentores y la revisión de los entregables intermedios. A lo largo del proceso de desarrollo de los proyectos, se suelen hacer dos tutorías plenarias en las que se explican las cuestiones comunes y transversales a todos los proyectos. Además, el/la tutor/a despeja las dudas específicas que pueden surgir en los distintos equipos, tanto en reuniones (presenciales 0 virtuales) 0 a través del correo electrónico. Entre sus principales funciones, podemos destacar las siguientes:

1. Definir la metodología, formato y calendario de entregables para la realización del proyecto.

2. Elaborar un sumario ejecutivo detallado para cada proyecto que recoge sus objetivos específicos.

3. Coordinar el equipo de mentores y facilitar su puesta en contacto con el correspondiente equipo de trabajo y representante de la entidad colaboradora.

4. Revisar los distintos entregables proporcionando correcciones y recomendaciones de mejora que los equipos deberán implementar de cara a cumplir con los objetivos mínimos y formato estipulado.

- Mentores: El mentor es un antiguo alumno con formación académica y/o experiencia profesional relevante para el proyecto práctico en cuestión. El mentor aporta su expertise, por sus conocimientos específicos y debido a su condición de alumni, para la buena realización del proyecto. Es decir, son facilitadores que ayudarán al equipo en el reparto de tareas, en alcanzar consensos, en la presentación del trabajo, en la resolución de cualquier cuestión disruptiva, entre otras. Esta figura no sustituye la labor de los alumnos, puesto que no es un 
miembro más del equipo ni tiene responsabilidad alguna sobre la ejecución del trabajo; ni tampoco la labor del tutor/a, puesto que no define metodologías, ni está en la obligación de corregir los entregables. Por tanto, la labor de los mentores se limita a contribuir a mejorar las dinámicas necesarias para la buena realización del trabajo grupal.

Las funciones de los mentores son las que siguen a continuación:

1. Centralizar y coordinar la comunicación entre el equipo y la entidad colaboradora (a través del representante designado a tal efecto). Es decir, el mentor se encargará de la interlocución con la entidad colaboradora a través de los canales acordados.

2. Orientar el trabajo del equipo. El mentor actúa como consejero o guía, que no ejecutor 0 tribunal, atendiendo a los objetivos, normas y metodología preestablecidos, y en base a sus conocimientos y experiencia. Cada equipo de trabajo acordará con el mentor las vías de comunicación y agendarán las reuniones que estimen convenientes, presencial o telemáticamente.

3. Asistir como mínimo a las dos tutorías plenarias, así como a las demás reuniones a las que sean convocados.

- Miembros del tribunal académico: Todos los proyectos prácticos se defienden ante un Comité científico constituido por $D^{\mathrm{a}}$. Araceli Torres, vicerrectora del campus de Ferrol y Responsabilidad Social; D. Antonio Abril, presidente del Consejo Social de la UDC; Da. Marta Rey, profesora contratada doctora de la UDC, directora académica del CESIS y directora de la Cátedra Inditex-UDC de Sostenibilidad; y D. Anxo Calvo, profesor titular de la UDC y decano de la Facultad de Economía y Empresa. Este acto de defensa de proyectos consiste en la exposición de cada uno de los equipos del trabajo realizado, haciendo especial hincapié en las principales conclusiones y recomendaciones, seguido de un turno de debate. A continuación, el tribunal juzga los proyectos y emite una calificación final de apto 0 no apto. A este acto público son invitados los representantes de las entidades colaboradoras, quienes participan activamente en el debate de sus respectivos proyectos, los mentores, el tutor/a y antiguos alumnos. 


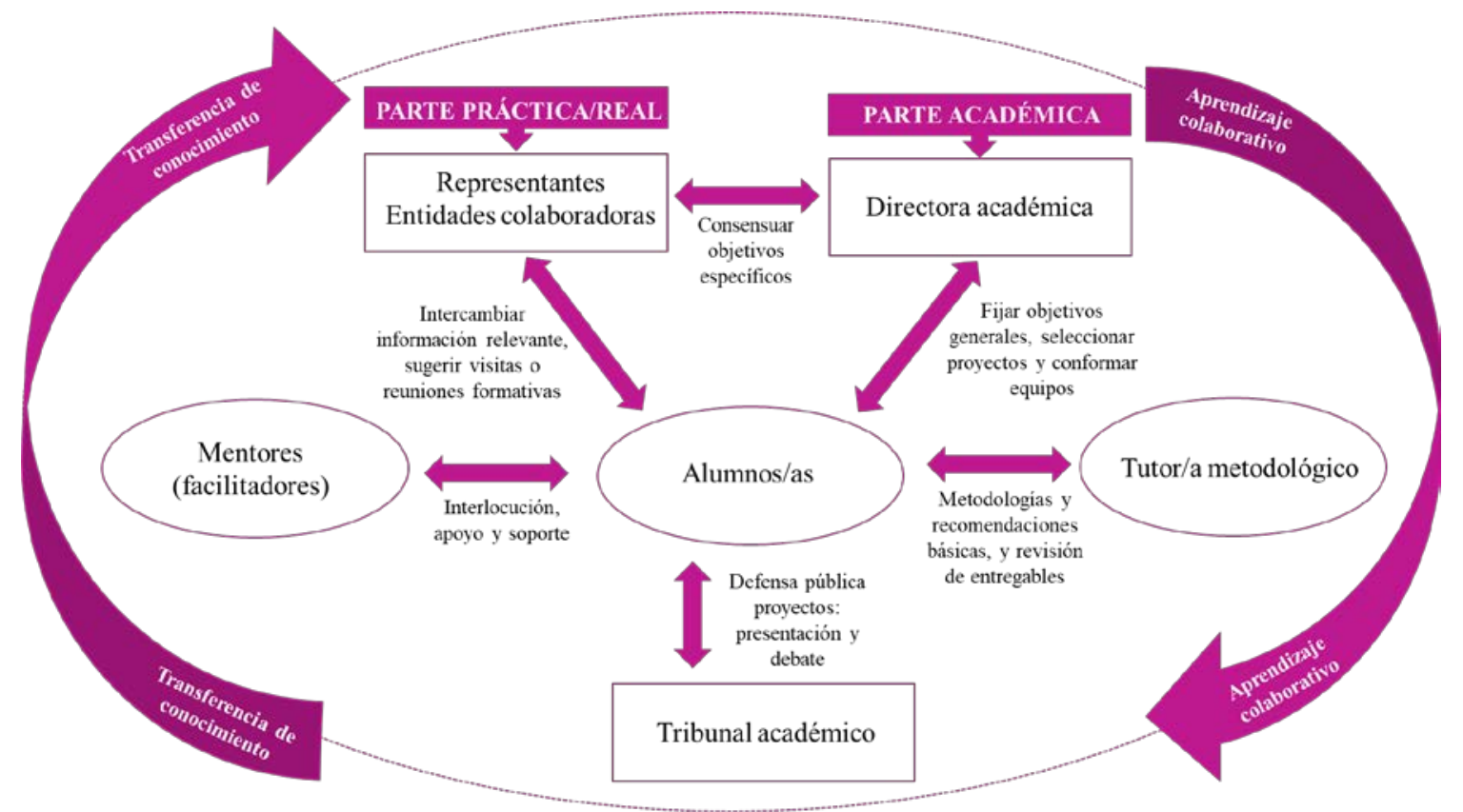

Figura 1. Relación entre los actores involucrados en los proyectos prácticos del CESIS.

Fuente: Elaboración propia

\section{RESULTADOS}

Para medir los resultados de los proyectos prácticos del CESIS, al término de cada edición los alumnos evalúan un conjunto de indicadores, lo que permite obtener el nivel de satisfacción de los alumnos con esta innovación educativa, a la vez que se valora su desempeño, tanto a nivel individual como de equipo. Asimismo, cada equipo también evalúa el desempeño de su mentor y del tutor. El objetivo de esta evaluación es someter esta metodología innovadora a un proceso de mejora continua, permitiendo a la dirección académica introducir las mejoras y ajustes necesarios para alcanzar el punto de equilibrio entre los requerimientos de aprendizaje de los alumnos y las expectativas de las entidades colaboradoras. La herramienta metodológica utilizada para la obtención de datos es un cuestionario online enviado a los alumnos a través de correo electrónico con escala Likert de cinco puntos (siendo 
$1=$ Deficiente, $\ldots 5=$ Excelente). La Figura 2 muestra los valores promedios resultado de todas las respuestas de los alumnos acerca del desempeño individual de cada uno de sus compañeros de equipo. Como podemos comprobar, durante los dos últimos cursos académicos, el resultado más bajo es de 4,46 (siendo $4=$ Muy buena y $5=$ Excelente), por lo que los estudiantes valoran muy positivamente el desempeño individual de sus compañeros. La tasa de respuesta del curso 2016/2017 es del 83,3\%, y del curso 2017/2018 es del 92,5\%.

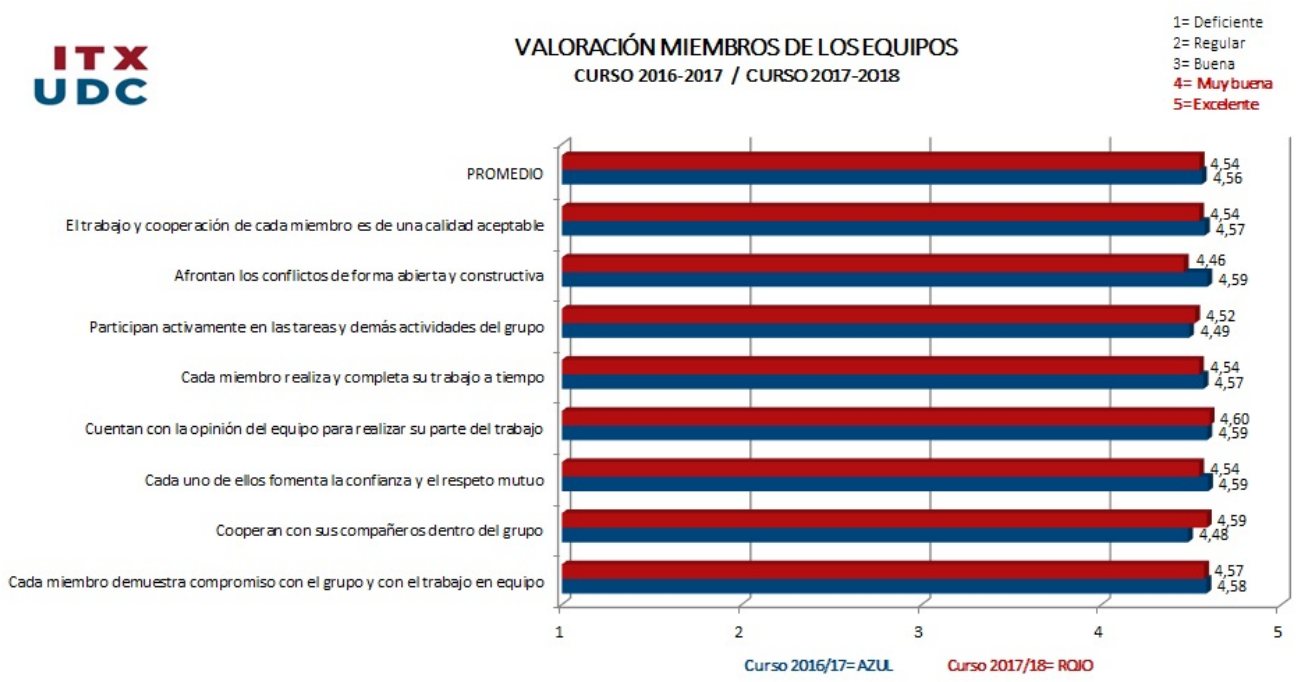

Figura 2. Valoración del desempeño individual de los miembros de los equipos de trabajo.

Fuente: Elaboración propia

A continuación, en la Figura 3 se exponen los valores promedios resultado de todas las respuestas de los estudiantes acerca del trabajo en equipo (si hay un reparto equitativo de tareas, si manejan adecuadamente los conflictos, etc.). En este caso, los valores son algo inferiores a los anteriores, lo que ratifica las dificultades que surgen a la hora de transformar el trabajo grupal en un verdadero trabajo en equipo. Pero, aun así, el valor más bajo se sitúa en 4,14 , es decir, todos los indicadores se encuentran nuevamente entre muy bueno y excelente. 


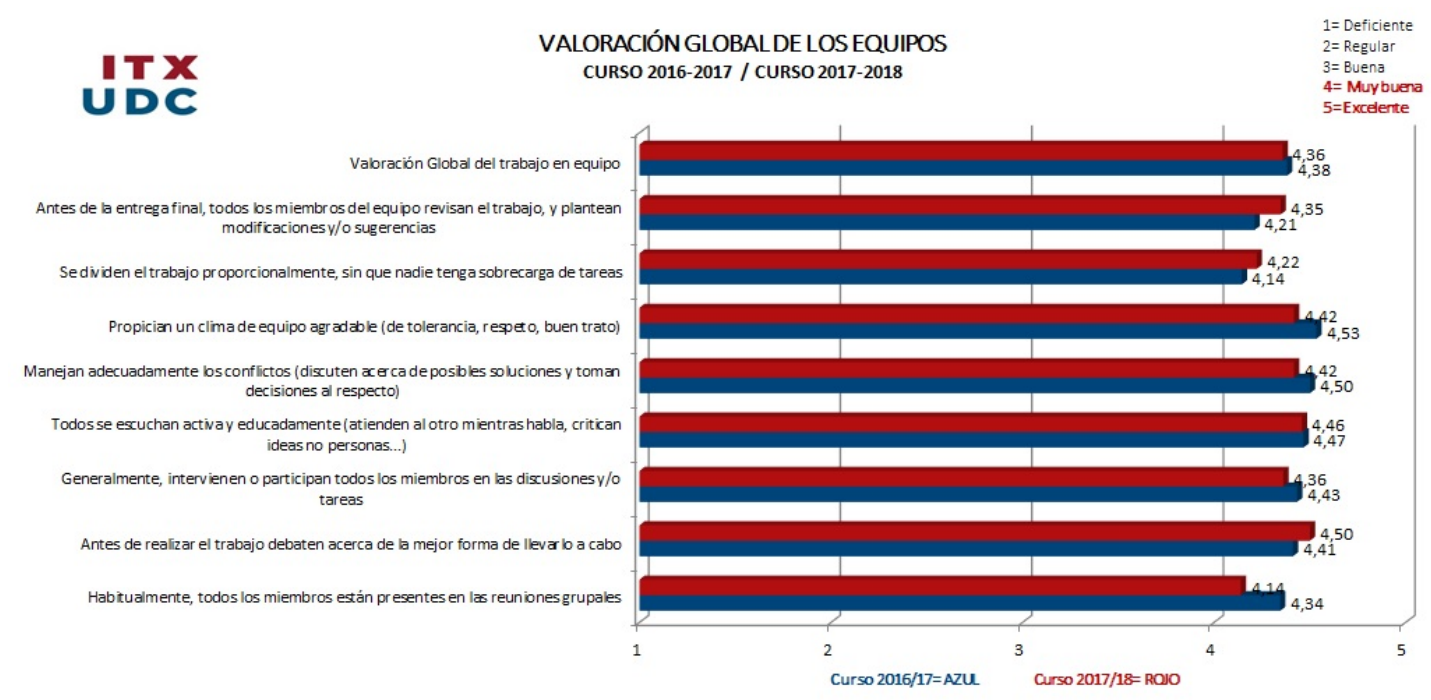

Figura 3. Valoración global del trabajo en equipo.

Fuente: Elaboración propia

Por tanto, en líneas generales, estos resultados muestran un elevado nivel de satisfacción de los estudiantes de las dos últimas ediciones con la realización de los proyectos en colaboración, en la medida en que las evaluaciones se sitúan entre los valores 4 "muy bueno" y 5 "excelente" (Figuras 2 y 3 ).

Finalmente, además de los resultados de aprendizaje y satisfacción de los estudiantes, también es importante destacar los resultados en cuanto a transferencia de conocimiento, esto es, en cuanto a implantación de las recomendaciones que se derivan de los proyectos en las entidades colaboradoras, o impacto de los proyectos sobre estas entidades. En este contexto, el concepto 'impacto' hace referencia a la relación causa-efecto establecida entre la ejecución de un programa o proyecto formativo, y el cambio o transformación producido por sus efectos en la población beneficiaria (Román, 2017). De esta forma se conjugan dos niveles de análisis: 1) la adquisición de nuevos conocimientos y competencias por parte de los alumnos, y nivel de aplicabilidad de lo aprendido; y 2) el desarrollo e implantación de recomendaciones y mejoras en las organizaciones (Tejada y Ferrández, 2007). Entre las recomendaciones de los 
proyectos que fueron implementadas por las entidades colaboradoras, podemos destacar algunos ejemplos: la elaboración y presentación de la primera memoria de sostenibilidad del Real Club Deportivo de A Coruña (año 2016); la organización de la primera Jornada de Responsabilidad Social en el ámbito sanitario en Galicia (organizada por la Gerencia de Gestión Integrada de Santiago de Compostela); o la creación de un sello para identificar el compromiso con los objetivos de la Reserva de la Biosfera "Mariñas Coruñesas e Terras do Mandeo" de las organizaciones localizadas en el área, considerando aspectos sociales, medioambientales y de buen gobierno.

\section{CONCLUSIONES}

A pesar de que los resultados alcanzados hasta el momento apuntan a un exitoso modelo de aprendizaje colaborativo para los alumnos (innovación educativa), y a la satisfacción de las entidades colaboradoras por obtener ideas y recomendaciones innovadoras para solucionar necesidades reales e inmediatas (transferencia de conocimiento), es necesario continuar aunando esfuerzos para abordar los retos que supone esta metodología. En particular, es necesario clarificar mejor a los estudiantes cómo deben aplicar los conocimientos adquiridos a la realidad de una organización, tratar de optimizar la coordinación y comunicación entre los distintos actores involucrados, dar más orientaciones sobre cómo desarrollar competencias que permitan trabajar en equipo, entre otras.

Finalmente, en futuras ediciones, además de averiguar el nivel de satisfacción, sería interesante realizar una verdadera evaluación de impacto que permitiera medir, en el medio 0 largo plazo, el impacto de esta innovación educativa en la trayectoria formativa y profesional de los alumnos y en el stock de conocimiento y práctica diaria de las entidades colaboradoras. 


\section{REFERENCIAS}

Espinosa, J.K., Jimenez, J., Olabe, M. \& Basogain, X. (2006). Innovación docente para el desarrollo de competencias en el EES. VII Congreso de Tecnologías Aplicadas a la Enseñanza de la Electrónica, Madrid.

Fernández, J. T. (2002). El docente universitario ante los nuevos escenarios: implicaciones para la innovación docente. Acción pedagógica, 11(2), pp. 30-42.

IESE (2018). El método del caso: una experiencia completa de aprendizaje: https://www.iese.edu/es/claustro-investigacion/metodo-del-caso/.

Naciones Unidas (2018). Objetivos de Desarrollo Sostenible:

https://www.un.org/sustainabledevelopment/es/objetivos-de-desarrollo-sostenible/.

Román, J. Y. G. (2017). La evaluación de impacto de programas educativos. Pedagogía Profesional, 15 (3), pp. 1-11.

Salido-Andrés, N., Felgueiras, A. \& Rey-García, M. (2014). Innovación docente en el marco de la colaboración Empresa - Universidad: La Cátedra Inditex de Responsabilidad Social de la Universidad de A Coruña. VI Workshop de Dirección Estratégica ACEDE, Oviedo, España.

Tejada, J. \& Ferrández, E. (2007). La evaluación del impacto de la formación como estrategia de mejora en las organizaciones. Revista electrónica de investigación educativa, 9(2), pp. 115.

UNESCO (2018). Education for Sustainable Development:

https://en.unesco.org/themes/education-sustainable-development-esd.

World Commission on Environment and Development [WCED] (1987). Report: Our Common Future:

http://www.exteriores.gob.es/Portal/es/PoliticaExteriorCooperacion/Desarrollosostenible/Docu ments/Informe\%20Brundtland\%20(En\%20ingl\%C3\%A9s).pdf. 